\title{
Solusi Persamaan Diferensial Fraksional Non-Linear Menggunakan Telescoping Decomposition Method
}

\author{
A Risara Vilinea ${ }^{1}$, E Rusyaman ${ }^{2}$, Eddy Djauhari ${ }^{3}$ \\ ${ }^{1}$ Program Studi S-1 Matematika Fakultas MIPA Universitas Padjadjaran, \\ anjang15001@mail.unpad.ac.id
}

\begin{abstract}
Abstrak
Model matematika banyak digunakan dalam memecahkan permasalahan, salah satunya persamaan diferensial. Pada umumnya persamaan diferensial menggunakan orde bilangan asli, namun orde pada persamaan diferensial dapat dibentuk menjadi orde pecahan yang disebut persamaan diferensial fraksional. Suatu persamaan diferensial fraksional dapat diselesaikan dan diperoleh solusinya. Ada beberapa metode yang dapat digunakan untuk menyelesaikan persamaan diferensial fraksional, salah satunya yaitu Telescoping Decomposition Method (TDM) yang merupakan modifikasi dari Adomian Decomposition Method (ADM). Penulis mencoba menyelesaikan persamaan diferensial fraksional non-linear menggunakan metode tersebut, dimana TDM lebih efisien dari ADM dalam mencari solusi persamaan diferensial fraksional non-linear. Selanjutnya, barisan orde dari persamaan diferensial fraksional non-linear dapat diamati kekonvergenannya ke suatu bilangan yang mengakibatkan barisan fungsi solusi dari persamaan diferensial fraksional non-linear akan konvergen ke fungsi solusi dengan orde bilangan itu sendiri dan akan dibandingkan hasilnya dengan Adomian Decomposition Method (ADM). Terbukti bahwa menggunakan TDM lebih cepat konvergen menuju solusi eksak dibandingkan dengan menggunkan ADM.

Kata kunci: Persamaan Diferensial, Fraksional, Non-linear, Telescoping Decomposition, Konvergen.
\end{abstract}

\begin{abstract}
Mathematical models are used in solving problems, one of which is differential equations. The differential equation generally uses a natural number order, but the order in the equation could be turned into a fraction order known as fractional differential equation, which could be solved. There are some methods that could be used to solve the fractional differential equation, one of which is the Telescoping Decomposition Method which is a modification of Adomian Decomposition Method $(A D M)$. In this study, The writer attempts to solve non-linear fractional differential equations using the method mentioned, where TDM is more efficient than ADM in finding solutions to non-linear fractional differential equations. Furthermore, the order sequence of a non-linear fractional differential equation convergences into a number, which is the process of solution function sequence converging from a non-linear fractional differential equation into a fractional differential equation with its own number order, could be observed and will compare the result with Adomian Decomposition Method. Proven that using TDM faster converges to exact solutions compared to using ADM.
\end{abstract}

2000 Mathematics Subject Classification: 26A33

Submitted: 08-07-2019, Revision: 11-01-2020, Accepted: 30-01-2020. 
Keywords: differential equation, fractional, non-linear, telescoping decomposition, convergent.

\section{PENDAHULUAN}

Persamaan diferensial adalah persamaan yang melibatkan turunan atau diferensial dari suatu fungsi yang tidak diketahui. Berdasarkan jumlah variabel bebasnya, persamaan diferensial terbagi menjadi dua jenis, yaitu Persamaan Diferensial Biasa (PDB) dan Persamaan Diferensial Parsial (PDP). Orde pada persamaan diferensial dapat dibentuk menjadi orde pecahan yang disebut persamaan diferensial fraksional. Salah satu tujuan mempelajari persamaan diferensial fraksional yaitu mencari penyelesaian atau solusi dari persamaan diferensial. Solusi persamaan diferensial fraksional adalah suatu fungsi yang memenuhi persamaan diferensial tersebut. Solusi dari persamaan diferensial fraksional dapat dicari dengan menggunakan beberapa metode, salah satunya Telescoping Decomposition Method (TDM). Beberapa tahun kebelakang banyak peneliti yang mengkaji tentang TDM sebagai metode yang digunakan dalam mencari solusi persamaan diferensial, seperti pada [1] telah menggunakan pada persamaan diferensial non-linear orde satu. Kemudian [2] menggunakan persamaan diferensial non-linear orde dua, serta pada [3] menggunakan TDM untuk mencari persamaan diferensial fraksional. Penulis menggunakan Telescoping Decomposition Method dalam mencari solusi persamaan diferensial non-linear berorde fraksional, kemudian akan dianalisis kekonvergenan barisan fungsi solusi, serta akan dibandingkan solusinya dengan menggunakan Adomian Decomposition Method.

\section{METODE PENELITIAN}

Pada bagian ini dibahas tentang pencarian solusi dari persamaan diferensial fraksional non-linear menggunakan Telescoping Decomposition Method (TDM) dengan bentuk umum persamaan diferensial fraksional non-linear yang digunakan adalah

$$
D^{\alpha} u(t)+A u^{2}(t)+B u(t)=r(t), 0<\alpha \leq 2, A, B \text { konstanta real }
$$

Telescoping Decomposition Method (TDM) adalah metode iteratif baru untuk persamaan diferensial fraksional non-linear. Dengan metode ini dapat dicari solusi numerik dari ekspansi deret Taylor yang mengkonstruksi solusi analitik dalam bentuk polinomial. Metode ini adalah bentuk modifikasi dari Adomian Decomposition Method (ADM), dimana pada TDM, polinomial Adomian tidak harus dihitung sehingga metode ini memberikan cara yang lebih sederhana dan menghasilkan pendekatan solusi ke nilai sebenarnya lebih efisien dari ADM, dengan rumus pendekatan solusi

$$
u(t)=\sum_{k=0}^{m} u_{k}(t) ; m \geq 1
$$

dimana $u_{k}(t)$ harus dicari secara berurutan melalui prosedur iteratif sebagai berikut,

$$
\begin{aligned}
& u_{0}(t)= \sum_{k=0}^{m-1} \frac{u^{(k)}\left(0^{+}\right)}{k !} t^{k} ; m=1 \\
& u_{1}(t)= \frac{1}{\Gamma(\alpha)} \int_{0}^{t}(t-s)^{\alpha-1} f\left(s, u_{0}(s)\right) d s=J^{\alpha}\left[f\left(t, u_{0}(t)\right)\right] \\
& u_{2}(t)= J^{\alpha}\left[f\left(t, u_{0}(t)+u_{1}(t)\right)\right]-J^{\alpha}\left[f\left(t, u_{0}(t)\right)\right] \\
& u_{3}(t)= J^{\alpha}\left[f\left(t, u_{0}(t)+u_{1}(t)+u_{2}(t)\right)\right]-J^{\alpha}\left[f\left(t, u_{0}(t)+u_{1}(t)\right)\right] \\
& \vdots \\
& u_{n}(t)=5 J^{\alpha}\left[f\left(t, \sum_{k=0}^{n-1} u_{k}(t)\right)\right]-J^{\alpha}\left[f\left(t, \sum_{k=0}^{n-2} u_{k}(t)\right)\right]
\end{aligned}
$$


Integral Fraksional didefinisikan oleh Riemann-Liouville sebagai berikut (Kisela,2008)

$$
J^{\alpha} f(x)=D_{\alpha}^{-\alpha} f(x)=\frac{1}{\Gamma(\alpha)} \int_{0}^{x}(x-t)^{\alpha-1} f(t) d t ; \alpha, x>0 .
$$

Teorema 2.1. Integral fraksional berorde $\alpha$ dari fungsi polinom sederhana yang berbentuk $f(x)=x^{n}$ adalah

$$
D^{-\alpha} f(x)=\frac{\Gamma(n+1)}{\Gamma(n+\alpha+1)} x^{n+\alpha} ; \alpha, x>0, n \geq 0 .
$$

Teorema 2.2. Misalkan $\alpha, \beta>0$ dan $\lambda, \mu$ merupakan konstanta, maka untuk integral fraksional berlaku sifat-sifat berikut:

$$
\begin{gathered}
J^{\alpha}(\lambda f(t)+\mu g(t))=\lambda J^{\alpha} f(t)+\mu J^{\alpha} g(t) . \\
J^{\alpha}\left(J^{-\beta} f(t)\right)=J^{\alpha-\beta} f(t .) \\
J^{\alpha}[\lambda \cdot f(t)]=\lambda \cdot J^{\alpha}[f(t)] .
\end{gathered}
$$

\section{HASIL DAN PEMBAHASAN}

3.1. Persamaan Diferensial Fraksional Orde $\alpha$ dengan $r(t)=k$. Diberikan persamaan diferensial fraksional non-linear berorde $\alpha$ berikut

$$
D^{\alpha} u(t)+A \cdot u^{2}(t)+B \cdot u(t)=k
$$

dengan kondisi awal $u(0)=0$.

Pencarian solusi dari persamaan diferensial fraksional berorde $\alpha$ menggunakan Telesoping Decomposition Method (TDM) sebagai berikut

$$
D^{\alpha} u(t)=f(t, u)=k-A \cdot u^{2}(t)-B \cdot u(t)
$$

Bentuk solusi pendekatan dari persamaan (7) dengan menggunakan langkah iteratif pada persamaan (2) hingga (6) sebagai berikut

$$
\begin{aligned}
u_{0}(t) & =0 \\
u_{1}(t) & =J^{\alpha}\left[f\left(t, u_{0}(t)\right)\right]=J^{\alpha}[k]=\frac{k t^{\alpha}}{\Gamma(\alpha+1)} \\
u_{2}(t) & =J^{\alpha}\left[f\left(t, u_{0}(t)+u_{1}(t)\right)\right]-J^{\alpha}\left[f\left(t, u_{0}(t)\right)\right] \\
& =J^{\alpha}\left\lceil k-A \cdot\left(\frac{k t^{\alpha}}{\Gamma(\alpha+1)}\right)^{2}-B \cdot \frac{k t^{\alpha}}{\Gamma(\alpha+1)}\right\rceil-J^{\alpha}[k] \\
& =-\frac{A k^{2} \Gamma(2 \alpha+1)}{\Gamma(\alpha+1)^{2} \Gamma(3 \alpha+1)} t^{3 \alpha}-\frac{B k}{\Gamma(2 \alpha+1)} t^{2 \alpha} \\
u_{3}(t) & =J^{\alpha}\left[f\left(t, u_{0}(t)+u_{1}(t)+u_{2}(t)\right)\right]-J^{\alpha}\left[f\left(t, u_{0}(t)+u_{1}(t)\right)\right] \\
& =\frac{B^{2} k}{\Gamma(2 \alpha+1)} \frac{\Gamma(2 \alpha+1)}{\Gamma(3 \alpha+1)} t^{3 \alpha}+\frac{2 A B k^{2}}{\Gamma(2 \alpha+1) \Gamma(\alpha+1)} \cdot \frac{\Gamma(3 \alpha+1)}{\Gamma(4 \alpha+1)} t^{4 \alpha} \\
& +\frac{A B k^{2} \cdot \Gamma(2 \alpha+1)}{\Gamma(2 \alpha+1)^{2} \Gamma(3 \alpha+1)} \cdot \frac{\Gamma(3 \alpha+1)}{\Gamma(4 \alpha+1)} t^{4 \alpha}-\frac{A B^{2} k^{2}}{\Gamma(2 \alpha+1)^{2}} \cdot \frac{\Gamma(4 \alpha+1)}{\Gamma(5 \alpha+1)} t^{5 \alpha} \\
& +\frac{2 A^{2} k^{3} \cdot \Gamma(2 \alpha+1)}{\Gamma(\alpha+1)^{3} \Gamma(3 \alpha+1)} \cdot \frac{\Gamma(4 \alpha+1)}{\Gamma(5 \alpha+1)} t^{5 \alpha}-\frac{2 A^{2} B k^{3}}{\Gamma(\alpha+1)^{2} \Gamma(3 \alpha+1)} \cdot \frac{\Gamma(5 \alpha+1)}{\Gamma(6 \alpha+1)} t^{6 \alpha} \\
& -\frac{A^{3} k^{4} \cdot \Gamma(2 \alpha+1)^{2}}{\Gamma(\alpha+1)^{4} \Gamma(3 \alpha+1)^{2}} \cdot \frac{\Gamma(6 \alpha+1)}{\Gamma(7 \alpha+1)} t^{7 \alpha} \cdot
\end{aligned}
$$


Dengan demikian diperoleh solusi pendekatan

$$
\begin{aligned}
u(t) & =\sum_{k=0}^{3} u_{k}(t) \\
& =\left(\frac{k t^{\alpha}}{\Gamma(\alpha+1)}\right)+\left(-\frac{A k^{2} \cdot \Gamma(2 \alpha+1)}{\Gamma(\alpha+1)^{2} \Gamma(3 \alpha+1)} t^{3 \alpha}-\frac{B k}{\Gamma(2 \alpha+1)} t^{2 \alpha}\right) \\
& +\frac{B^{2} k}{\Gamma(2 \alpha+1)} \cdot \frac{\Gamma(2 \alpha+1)}{\Gamma(3 \alpha+1)} t^{3 \alpha}+\frac{2 A B k^{2}}{\Gamma(2 \alpha+1) \Gamma(\alpha+1)} \cdot \frac{\Gamma(3 \alpha+1)}{\Gamma(4 \alpha+1)} t^{4 \alpha} \\
& +\frac{A B k^{2} \cdot \Gamma(2 \alpha+1)}{\Gamma(\alpha+1)^{2} \Gamma(3 \alpha+1)} \cdot \frac{\Gamma(3 \alpha+1)}{\Gamma(4 \alpha+1)} t^{4 \alpha}-\frac{A B^{2} k^{2}}{\Gamma(2 \alpha+1)^{2}} \cdot \frac{\Gamma(4 \alpha+1)}{\Gamma(5 \alpha+1)} t^{5 \alpha} \\
& +\frac{2 A^{2} k^{3} \cdot \Gamma(2 \alpha+1)}{\Gamma(\alpha+1)^{3} \Gamma(3 \alpha+1)} \cdot \frac{\Gamma(4 \alpha+1)}{\Gamma(3 \alpha+1)} t^{5 \alpha}-\frac{2 A^{2} B k^{3}}{\Gamma(\alpha+1)^{2} \Gamma(3 \alpha+1)} \cdot \frac{A^{3}(5 \alpha+1)}{\Gamma(6 \alpha+1)} t^{6 \alpha} \\
& -\frac{A^{3} k^{4} \cdot \Gamma(2 \alpha+1)^{2}}{\Gamma(\alpha+1)^{4} \Gamma(3 \alpha+1)^{2}} \cdot \frac{\Gamma(6 \alpha+1)}{\Gamma(7 \alpha+1)} t^{7 \alpha} .
\end{aligned}
$$

selanjutnya akan dicari solusi dari persamaan (7) dengan $A=1, B=0, k=1$.

$$
D^{\alpha} u(t)+u^{2}(t)=1
$$

dengan kondisi awal $u(0)=0$, diperoleh solusi pendekatan persamaan (8) sebagai berikut

$$
\begin{aligned}
u(t) & =\frac{t^{\alpha}}{\Gamma(\alpha+1)}-\frac{\Gamma(2 \alpha+1)}{\Gamma(\alpha+1)^{2} \Gamma(3 \alpha+1)} t^{3 \alpha}+\frac{2 \Gamma(2 \alpha+1)}{\Gamma(3 \alpha+1) \Gamma(\alpha+1)^{3}} \cdot \frac{\Gamma(4 \alpha+1)}{\Gamma(5 \alpha+1)} t^{5 \alpha} \\
& -\frac{\Gamma(2 \alpha+1)^{2}}{\Gamma(3 \alpha+1)^{2} \Gamma(\alpha+1)^{4}} \cdot \frac{\Gamma(6 \alpha+1)}{\Gamma(7 \alpha+1)} t^{7 \alpha}
\end{aligned}
$$

Selanjutnya kita akan melihat kekonvergenan barisan fungsi solusi persamaan diferensial fraksioal orde $\alpha_{n}$ dengan mengambil suatu barisan $\left(\alpha_{n}\right)=\left(\frac{n}{n+1}\right)$, maka persamaan diferensial fraksional orde $\alpha_{n}$ menjadi

$$
D^{\frac{n}{n+1}} u(t)+u^{2}(t)=1, n \in \mathbb{N}
$$

sehingga diperoleh barisan fungsi solusi persamaan diferensial fraksional berorde $\alpha_{n}$ yang dinotasikan $u_{n}(t)$ sebagai berikut,

$$
\begin{aligned}
u_{n}(t) & =\frac{t^{\frac{n}{n+1}}}{\Gamma\left(\frac{n}{n+1}+1\right)}-\frac{\Gamma\left(2 \cdot \frac{n}{n+1}+1\right) t^{3 \frac{n}{n+1}}}{\Gamma\left(\frac{n}{n+1}+1\right)^{2} \Gamma\left(3 \cdot \frac{n}{n+1}+1\right)}+\frac{2 \Gamma\left(2 \cdot \frac{n}{n+1}+1\right) \Gamma\left(4 \cdot \frac{n}{n+1}+1\right) t^{5 \cdot \frac{n}{n+1}}}{\Gamma\left(3 \cdot \frac{n}{n+1}+1\right) \Gamma\left(\frac{n}{n+1}+1\right)^{3} \Gamma\left(5 \cdot \frac{n}{n+1}+1\right)} \\
& -\frac{\Gamma\left(2 \cdot \frac{n}{n+1}+1\right)^{2}}{\Gamma\left(3 \cdot \frac{n}{n+1}+1\right)^{2} \Gamma\left(\frac{n}{n+1}+1\right)^{4}} \cdot \frac{\Gamma\left(6 \cdot \frac{n}{n+1}+1\right)}{\Gamma\left(7 \cdot \frac{n}{n+1}+1\right)} t^{7 \cdot \frac{n}{n+1}}
\end{aligned}
$$

Selanjutnya akan diperlihatkan barisan fungsi $\left(u_{n}(t)\right)$ konvergen ke fungsi $u(t)$, dimana $u(t)$ adalah solusi persamaan diferensial fraksional non-linear orde $\alpha=1$.

$$
u(t)=t-\frac{t^{3}}{3}+\frac{2 t^{5}}{5}-\frac{t^{7}}{63}
$$




$$
\begin{aligned}
\lim _{n \rightarrow \infty} u_{n}(t) & =\lim _{n \rightarrow \infty} \frac{t^{\frac{n}{n+1}}}{\Gamma\left(\frac{n}{n+1}+1\right)}-\frac{\Gamma\left(2 \cdot \frac{n}{n+1}+1\right) t^{3 \frac{n}{n+1}}}{\Gamma\left(\frac{n}{n+1}+1\right)^{2} \Gamma\left(3 \cdot \frac{n}{n+1}+1\right)}+\frac{2 \Gamma\left(2 \cdot \frac{n}{n+1}+1\right) \Gamma\left(4 \cdot \frac{n}{n+1}+1\right) t^{5 \cdot \frac{n}{n+1}}}{\Gamma\left(3 \cdot \frac{n}{n+1}+1\right) \Gamma\left(\frac{n}{n+1}+1\right)^{3} \Gamma\left(5 \cdot \frac{n}{n+1}+1\right)} \\
& -\frac{\Gamma\left(2 \cdot \frac{n}{n+1}+1\right)^{2}}{\Gamma\left(3 \cdot \frac{n}{n+1}+1\right)^{2} \Gamma\left(\frac{n}{n+1}+1\right)^{4}} \cdot \frac{\Gamma\left(6 \cdot \frac{n}{n+1}+1\right)}{\Gamma\left(7 \cdot \frac{n}{n+1}+1\right)} t^{7 \cdot \frac{n}{n+1}} \\
& =t-\frac{t^{3}}{3}+\frac{2 t^{5}}{5}-\frac{t^{7}}{63} \\
& =u(t)
\end{aligned}
$$

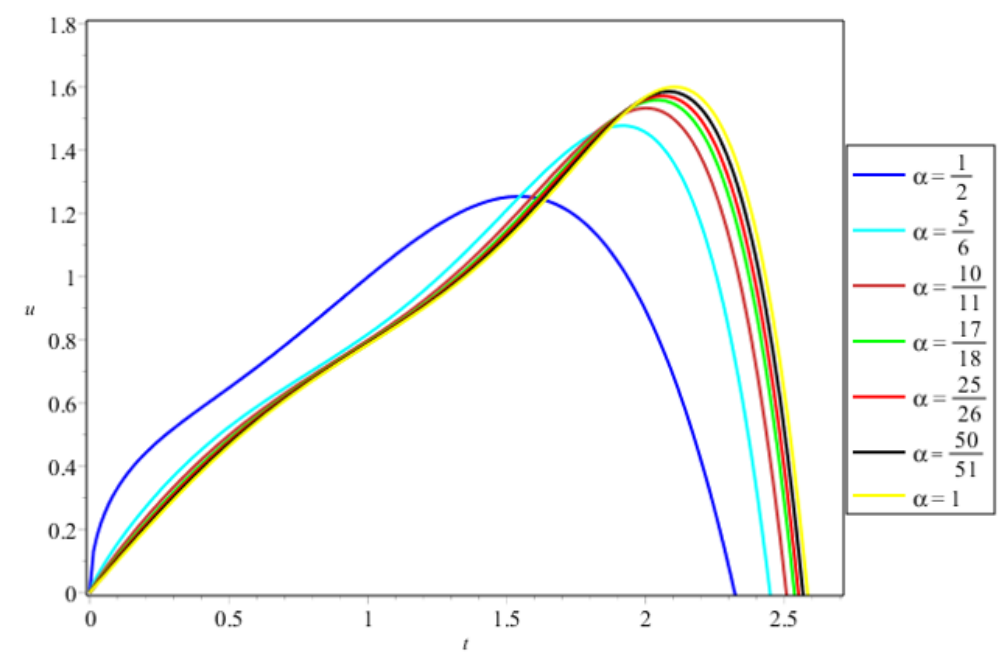

GAmBAR 1. Grafik fungsi solusi $D^{\alpha} u(t)+u^{2}(t)=1$ orde $\left(\alpha_{n}\right)=\left(\frac{n}{n+1}\right)$

Berdasarkan Gambar 1, solusi dikerjakan sampai iterasi suku ke-3, dapat dilihat bahwa persamaan diferensial fraksional non-linear dengan berorde $\left(\alpha_{n}\right)$ menghasilkan pola grafik yang bergerak menuju persamaan diferensial fraksional berorde $\alpha=1$, sehingga barisan fungsi solusi persamaan diferensial fraksional non-linear dengan bentuk $D^{\left(\frac{n}{n+1}\right)} u(t)+u^{2}(t)=1$ konvergen ke fungsi solusi persamaan diferensial fraksional non-linear dengan bentuk $D^{1} u(t)+u^{2}(t)=1$.

3.2. Persamaan Diferensial Fraksional Orde $\alpha$ dengan $r(t)=e^{k t}$. Diberikan persamaan diferensial fraksional non-linear berorde $\alpha$ berikut

$$
D^{\alpha} u(t)=f(t, u)=e^{k t}-A \cdot u^{2}(t)+B \cdot u(t)=e^{k t}
$$

dengan kondisi awal $u(0)=0$ 
Metode pencarian solusi dari persamaan diferensial fraksional berorde $\alpha$ menggunakan Telesoping Decomposition Method (TDM) sebagai berikut

$u_{0}(t)=0$.

$$
\begin{aligned}
u_{1}(t) & =J^{\alpha} f\left(t, u_{0}(t)\right)=J^{\alpha}\left[e^{k t}\right]=J^{\alpha}\left[1+\frac{k t}{1 !}+\frac{k^{2} t^{2}}{2 !}+\frac{k^{3} t^{3}}{3 !}+\ldots\right] \\
u_{2}(t) & =J^{\alpha}\left[f\left(t, u_{0}(t)+u_{1}(t)\right)\right]-J^{\alpha} f\left(t, u_{0}(t)\right) \\
& =-A \cdot\left(\sum_{m=0}^{3} \frac{k^{2 m} t^{3 \alpha+2 m}}{\Gamma(\alpha+m+1)^{2}} \frac{\Gamma(2 \alpha+2 m+1)}{\Gamma(3 \alpha+2 m+1)}\right)-A \cdot\left(\sum_{m=1}^{3} \frac{2 k^{m} t^{3 \alpha+m}}{\Gamma(\alpha+1) \Gamma(\alpha+m+1)} \frac{\Gamma(2 \alpha+m+1)}{\Gamma(3 \alpha+m+1)}\right) \\
& -A \cdot\left(\sum_{m=3}^{4} \frac{2 k^{m} t^{3 \alpha+m}}{\Gamma(\alpha+2) \Gamma(\alpha+m)} \frac{\Gamma(2 \alpha+m+1)}{\Gamma(3 \alpha+m+1)}\right)-A \cdot \frac{2 k^{5} t^{3 \alpha+5}}{\Gamma(\alpha+3) \Gamma(\alpha+4)} \frac{\Gamma(2 \alpha+6)}{\Gamma(3 \alpha+6)} \\
& -B \cdot\left(\sum_{m=0}^{3} \frac{k^{m} t^{2 \alpha+m}}{\Gamma(2 \alpha+m+1)}\right)+\ldots
\end{aligned}
$$

Dengan demikian diperoleh solusi pendekatan

$$
\begin{aligned}
u(t) & =\sum_{k=0}^{2} u_{k}(t) \\
& =\sum_{m=0}^{3} \frac{k^{m} t^{\alpha+m}}{\Gamma(\alpha+m+1)}-A \cdot\left(\sum_{m=0}^{3} \frac{k^{2 m} t^{3 \alpha+2 m}}{\Gamma(\alpha+m+1)^{2}} \frac{\Gamma(2 \alpha+2 m+1)}{\Gamma(3 \alpha+2 m+1)}\right) \\
& -A \cdot\left(\sum_{m=1}^{3} \frac{2 k^{m} t^{3 \alpha+m}}{\Gamma(\alpha+1) \Gamma(\alpha+m+1)} \frac{\Gamma(2 \alpha+m+1)}{\Gamma(3 \alpha+m+1)}\right)-A \cdot\left(\sum_{m=3}^{4} \frac{2 k^{m} t^{3 \alpha+m}}{\Gamma(\alpha+2) \Gamma(\alpha+m)} \frac{\Gamma(2 \alpha+m+1)}{\Gamma(3 \alpha+m+1)}\right) \\
& -A \cdot \frac{2 k^{5} t^{3 \alpha+5}}{\Gamma(\alpha+3) \Gamma(\alpha+4)} \frac{\Gamma(2 \alpha+6)}{\Gamma(3 \alpha+6)}-B \cdot\left(\sum_{m=0}^{3} \frac{k^{m} t^{2 \alpha+m}}{\Gamma(2 \alpha+m+1)}\right)+\ldots
\end{aligned}
$$

selanjutnya akan dicari solusi dari persamaan (9) dengan $A=1, B=1, k=3$,maka persamaan diferensial fraksional non-linear berorde $\alpha$ sebagai berikut

$$
D^{\alpha} u(t)+u^{2}(t)+u(t)=e^{3 t}
$$

dengan kondisi awal $u(0)=0$, diperoleh solusi pendekatan persamaan (10) sebagai berikut

$$
\begin{aligned}
u(t) & =\frac{t^{\alpha}}{\Gamma(\alpha+1)}+\frac{3}{\Gamma(\alpha+2)} t^{\alpha+1}+\frac{3^{2}}{\Gamma(\alpha+3)} t^{\alpha+2}+\frac{3^{3}}{\Gamma(\alpha+4)} t^{\alpha+3}-\frac{1}{\Gamma(\alpha+1)^{2}} \frac{\Gamma(2 \alpha+1)}{\Gamma(3 \alpha+1)} t^{3 \alpha} \\
& -\frac{6}{\Gamma(\alpha+1) \Gamma(\alpha+2)} \frac{\Gamma(2 \alpha+2)}{\Gamma(3 \alpha+2)} t^{3 \alpha+1}-\frac{18}{\Gamma(\alpha+1) \Gamma(\alpha+3)} \frac{\Gamma(2 \alpha+3)}{\Gamma(3 \alpha+3)} t^{3 \alpha+2} \\
& -\frac{54}{\Gamma(\alpha+1) \Gamma(\alpha+2)} \frac{\Gamma(2 \alpha+4)}{\Gamma(3 \alpha+4)} t^{3 \alpha+3}-\frac{9}{\Gamma(\alpha+2)^{2}} \frac{\Gamma(2 \alpha+3)}{\Gamma(3 \alpha+3)} t^{3 \alpha+2}-\frac{54}{\Gamma(\alpha+2) \Gamma(\alpha+3)} \frac{\Gamma(2 \alpha+4)}{\Gamma(3 \alpha+4)} t^{3 \alpha+3} \\
& -\frac{162}{\Gamma(\alpha+2) \Gamma(\alpha+4)} \frac{\Gamma(2 \alpha+5)}{\Gamma(3 \alpha+5)} t^{3 \alpha+4}-\frac{81}{\Gamma(\alpha+3)^{2}} \frac{\Gamma(2 \alpha+5)}{\Gamma(3 \alpha+5)} t^{3 \alpha+4}-\frac{2 \alpha+6}{\Gamma(\alpha+3) \Gamma(\alpha+4)} \frac{1}{\Gamma(3 \alpha+6)} t^{3 \alpha+5} \\
& -\frac{729}{\Gamma(\alpha+4)^{2}} \frac{\Gamma(2 \alpha+7)}{\Gamma(3 \alpha+7)} t^{3 \alpha+6}-\frac{1}{\Gamma(\alpha+1)} \frac{\Gamma(\alpha+1)}{\Gamma(2 \alpha+1)} t^{2 \alpha}-\frac{3}{\Gamma(\alpha+2)} \frac{\Gamma(2 \alpha+2)}{\Gamma(\alpha+1} t^{2 \alpha+1} \\
& -\frac{9}{\Gamma(\alpha+3)} \frac{\Gamma(\alpha+3)}{\Gamma(2 \alpha+3)} t^{2 \alpha+2}-\frac{27}{\Gamma(\alpha+4)} \frac{\Gamma(\alpha+4)}{\Gamma(2 \alpha+4)} t^{2 \alpha+3}+\ldots
\end{aligned}
$$

Selanjutnya kita akan melihat kekonvergenan barisan fungsi solusi persamaan diferensial fraksioal orde $\alpha_{n}$ dengan mengambil suatu barisan $\left(\alpha_{n}\right)=\frac{3 n}{4 n+1}$, maka persamaan diferensial fraksional orde $\alpha_{n}$ menjadi

$$
D^{\left(\frac{3 n}{4 n+1}\right)} u(t)+u^{2}(t)+u(t)=e^{3 t}, n \in \mathbb{N} .
$$


Sehingga diperoleh barisan fungsi solusi persamaan diferensial fraksional berorde $\alpha_{n}$ yang dinotasikan $u_{n}(t)$ sebagai berikut

$$
\begin{aligned}
& u_{n}(t)=\frac{t^{\frac{3 n}{4 n+1}}}{\Gamma\left(\frac{3 n}{4 n+1}+1\right)}+\frac{3 t^{\frac{3 n}{4 n+1}}+2}{\Gamma\left(\frac{3 n}{4 n+1}+2\right)}+\frac{9 t^{\frac{3 n}{4 n+1}+2}}{\Gamma\left(\frac{3 n}{4 n+1}+3\right)}+\frac{27 t^{\frac{3 n}{4 n+1}}+3}{\Gamma\left(\frac{3 n}{4 n+1}+4\right)} \\
& -\frac{\Gamma(2+1) t^{3 \cdot \frac{3 n}{4 n+1}}}{\Gamma\left(\frac{3 n}{4 n+1}+1\right)^{2} \Gamma\left(3 \cdot \frac{3 n}{4 n+1}+1\right)}-\frac{6 \Gamma\left(2 \cdot \frac{3 n}{4 n+1}+2\right) t^{3 \frac{3 n}{4 n+1}+1}}{\Gamma\left(\frac{3 n}{4 n+1}+1\right) \Gamma\left(\frac{3 n}{4 n+1}+2\right) \Gamma\left(3 \cdot \frac{3 n}{4 n+1}+2\right)} \\
& -\frac{18 \Gamma\left(2 \cdot \frac{3 n}{4 n+1}+3\right) t^{3 \frac{3 n}{4 n+1}+2}}{\Gamma\left(\frac{3 n}{4 n+1}+1\right) \Gamma\left(\frac{3 n}{4 n+1}+3\right) \Gamma\left(3 \cdot \frac{3 n}{4 n+1}+3\right)}-\frac{54 \Gamma\left(2 \cdot \frac{3 n}{4 n+1}+4\right) t^{3 \cdot \frac{3 n}{4 n+1}+3}}{\Gamma\left(\frac{e n}{4 n+1}+1\right) \Gamma\left(\frac{3 n}{4 n+1}+4\right) \Gamma\left(3 \cdot \frac{3 n}{4 n+1}+4\right)} \\
& -\frac{9 \Gamma\left(2 \cdot \frac{3 n}{4 n+1}+3\right) t^{3 \cdot \frac{3 n}{4 n+1}+2}}{\Gamma\left(\frac{3 n}{4 n+1}+2\right)^{2} \Gamma\left(3 \cdot \frac{3 n}{4 n+1}+3\right)}-\frac{54 \Gamma\left(2 \cdot \frac{3 n}{4 n+1}+4\right) t^{3 \cdot \frac{3 n}{4 n+1}+3}}{\Gamma\left(\frac{3 n}{4 n+1}+2\right) \Gamma\left(\frac{3 n}{4 n+1}+3\right) \Gamma\left(3 \cdot \frac{3 n}{4 n+1}+4\right)} \\
& -\frac{162 \Gamma\left(2 \cdot \frac{3 n}{4 n+1}+5\right) t^{3 \frac{3 n}{4 n+1}+4}}{\Gamma\left(\frac{3 n}{4 n+1}+2\right) \Gamma\left(\frac{3 n}{4 n+1}+4\right) \Gamma\left(3 \cdot \frac{3 n}{4 n+1}+5\right)}-\frac{81 \Gamma\left(2 \cdot \frac{3 n}{4 n+1}+5\right) t^{\frac{3 n}{4 n+1}+4}}{\Gamma\left(\frac{3 n}{4 n+1}+3\right)^{2} \Gamma\left(3 \cdot \frac{3 n}{4 n+1}+5\right)} \\
& -\frac{486 \Gamma\left(2 \cdot \frac{3 n}{4 n+1}+6\right) t^{3 \frac{3 n}{4 n+1}+5}}{\Gamma\left(\frac{3 n}{4 n+1}+3\right) \Gamma\left(\frac{3 n}{4 n+1}+4\right) \Gamma\left(3 \cdot \frac{3 n}{4 n+1}+6\right)}-\frac{729 \Gamma\left(2 \cdot \frac{3 n}{4 n+1}+7\right) t^{3 \frac{3 n}{4 n+1}+6}}{\Gamma\left(\frac{3 n}{4 n+1}+4\right)^{2} \Gamma\left(3 \cdot \frac{3 n}{4 n+1}+7\right)} \\
& -\frac{\Gamma\left(\frac{3 n}{4 n+1}+1\right) t^{2 \frac{3 n}{4 n+1}}}{\Gamma\left(\frac{3 n}{4 n+1}+1\right) \Gamma\left(2 \cdot \frac{3 n}{4 n+1}+1\right)}-\frac{3 \Gamma\left(\frac{3 n}{4 n+1}+2\right) t^{2 \frac{3 n}{4 n+1}+1}}{\Gamma\left(\frac{3 n}{4 n+1}+2\right) \Gamma\left(2 \cdot \frac{3 n}{4 n+1}+2\right)} \\
& -\frac{9 \Gamma\left(\frac{3 n}{4 n+1}+3\right) t^{2 \frac{3 n}{4 n+1}+2}}{\Gamma\left(\frac{3 n}{4 n+1}+3\right) \Gamma\left(2 \cdot \frac{3 n}{4 n+1}+3\right)}-\frac{27 \Gamma\left(\frac{3 n}{4 n+1}+4\right) t^{2 \frac{3 n}{4 n+1}+3}}{\Gamma\left(\frac{3 n}{4 n+1}+4\right) \Gamma\left(2 \cdot \frac{3 n}{4 n+1}+4\right)}+\ldots
\end{aligned}
$$

Selanjutnya akan diperlihatkan barisan fungsi $\left(u_{n}(t)\right)$ konvergen ke fungsi $u(t)$, dimana $u(t)$ adalah solusi PDF non-linear orde $\alpha=\frac{3}{4}$.

$$
\begin{aligned}
u(t) & =\left(\frac{1}{\Gamma\left(\frac{3}{4}\right)}\left(\frac{4}{3} t^{\frac{3}{4}}+\frac{16}{7} t^{\frac{7}{4}}+\frac{192}{77} t^{\frac{11}{4}}+\frac{768}{385} t^{\frac{15}{4}}\right)\right)+\left(-\frac{1}{\sqrt{\pi}}\left(\frac{4 t^{\frac{3}{2}}}{3}+\frac{8 t^{\frac{5}{2}}}{5}+\frac{48 t^{\frac{7}{2}}}{35}+\frac{32 t^{\frac{9}{2}}}{35}\right)\right) \\
& -\frac{\sqrt{\pi}}{\Gamma\left(\frac{3}{4}\right)^{2} \Gamma\left(\frac{1}{4}\right)}\left(\frac{256 t^{\frac{9}{4}}}{45}+\frac{4096 t^{\frac{13}{4}}}{819}+\frac{373760 t^{\text {fra } 21}}{51051}+\frac{524288 t^{\frac{21}{4}}}{54145}+\frac{116391936 t^{\frac{25}{4}}}{14889875}\right) \\
& -\frac{\sqrt{\pi}}{\Gamma\left(\frac{3}{4}\right)^{2} \Gamma\left(\frac{1}{4}\right)}\left(\frac{1509949446 t^{\frac{29}{4}}}{33215875}+\frac{603976776 t^{\frac{33}{4}}}{365374625}\right)+\ldots
\end{aligned}
$$




$$
\begin{aligned}
& \lim _{n \rightarrow \infty} u_{n}(t)=\lim _{n \rightarrow \infty} \frac{t^{\frac{3 n}{4 n+1}}}{\Gamma\left(\frac{3 n}{4 n+1}+1\right)}+\frac{3 t^{\frac{3 n}{4 n+1}}+2}{\Gamma\left(\frac{3 n}{4 n+1}+2\right)}+\frac{9 t^{\frac{3 n}{4 n+1}}+2}{\Gamma\left(\frac{3 n}{4 n+1}+3\right)}+\frac{27 t^{\frac{3 n}{4 n+1}}+3}{\Gamma\left(\frac{3 n}{4 n+1}+4\right)} \\
& -\frac{\Gamma(2+1) t^{3 \cdot \frac{3 n}{4 n+1}}}{\Gamma\left(\frac{3 n}{4 n+1}+1\right)^{2} \Gamma\left(3 \cdot \frac{3 n}{4 n+1}+1\right)}-\frac{6 \Gamma\left(2 \cdot \frac{3 n}{4 n+1}+2\right) t^{3 \frac{3 n}{4 n+1}+1}}{\Gamma\left(\frac{3 n}{4 n+1}+1\right) \Gamma\left(\frac{3 n}{4 n+1}+2\right) \Gamma\left(3 \cdot \frac{3 n}{4 n+1}+2\right)} \\
& -\frac{18 \Gamma\left(2 \cdot \frac{3 n}{4 n+1}+3\right) t^{3 \frac{3 n}{4 n+1}+2}}{\Gamma\left(\frac{3 n}{4 n+1}+1\right) \Gamma\left(\frac{3 n}{4 n+1}+3\right) \Gamma\left(3 \cdot \frac{3 n}{4 n+1}+3\right)}-\frac{54 \Gamma\left(2 \cdot \frac{3 n}{4 n+1}+4\right) t^{3 \cdot \frac{3 n}{4 n+1}+3}}{\Gamma\left(\frac{e n}{4 n+1}+1\right) \Gamma\left(\frac{3 n}{4 n+1}+4\right) \Gamma\left(3 \cdot \frac{3 n}{4 n+1}+4\right)} \\
& -\frac{9 \Gamma\left(2 \cdot \frac{3 n}{4 n+1}+3\right) t^{3 \cdot \frac{3 n}{4 n+1}+2}}{\Gamma\left(\frac{3 n}{4 n+1}+2\right)^{2} \Gamma\left(3 \cdot \frac{3 n}{4 n+1}+3\right)}-\frac{54 \Gamma\left(2 \cdot \frac{3 n}{4 n+1}+4\right) t^{3 \cdot \frac{3 n}{4 n+1}+3}}{\Gamma\left(\frac{3 n}{4 n+1}+2\right) \Gamma\left(\frac{3 n}{4 n+1}+3\right) \Gamma\left(3 \cdot \frac{3 n}{4 n+1}+4\right)} \\
& -\frac{162 \Gamma\left(2 \cdot \frac{3 n}{4 n+1}+5\right) t^{3 \frac{3 n}{4 n+1}+4}}{\Gamma\left(\frac{3 n}{4 n+1}+2\right) \Gamma\left(\frac{3 n}{4 n+1}+4\right) \Gamma\left(3 \cdot \frac{3 n}{4 n+1}+5\right)}-\frac{81 \Gamma\left(2 \cdot \frac{3 n}{4 n+1}+5\right) t^{3 \frac{3 n}{4 n+1}+4}}{\Gamma\left(\frac{3 n}{4 n+1}+3\right)^{2} \Gamma\left(3 \cdot \frac{3 n}{4 n+1}+5\right)} \\
& -\frac{486 \Gamma\left(2 \cdot \frac{3 n}{4 n+1}+6\right) t^{3 \frac{3 n}{4 n+1}+5}}{\Gamma\left(\frac{3 n}{4 n+1}+3\right) \Gamma\left(\frac{3 n}{4 n+1}+4\right) \Gamma\left(3 \cdot \frac{3 n}{4 n+1}+6\right)}-\frac{729 \Gamma\left(2 \cdot \frac{3 n}{4 n+1}+7\right) t^{3 \frac{3 n}{4 n+1}+6}}{\Gamma\left(\frac{3 n}{4 n+1}+4\right)^{2} \Gamma\left(3 \cdot \frac{3 n}{4 n+1}+7\right)} \\
& -\frac{\Gamma\left(\frac{3 n}{4 n+1}+1\right) t^{2 \frac{3 n}{4 n+1}}}{\Gamma\left(\frac{3 n}{4 n+1}+1\right) \Gamma\left(2 \cdot \frac{3 n}{4 n+1}+1\right)}-\frac{3 \Gamma\left(\frac{3 n}{4 n+1}+2\right) t^{2 \frac{3 n}{4 n+1}+1}}{\Gamma\left(\frac{3 n}{4 n+1}+2\right) \Gamma\left(2 \cdot \frac{3 n}{4 n+1}+2\right)} \\
& -\frac{9 \Gamma\left(\frac{3 n}{4 n+1}+3\right) t^{2 \frac{3 n}{4 n+1}+2}}{\Gamma\left(\frac{3 n}{4 n+1}+3\right) \Gamma\left(2 \cdot \frac{3 n}{4 n+1}+3\right)}-\frac{27 \Gamma\left(\frac{3 n}{4 n+1}+4\right) t^{2 \frac{3 n}{4 n+1}+3}}{\Gamma\left(\frac{3 n}{4 n+1}+4\right) \Gamma\left(2 \cdot \frac{3 n}{4 n+1}+4\right)}+\ldots \\
& =\left(\frac{1}{\Gamma\left(\frac{3}{4}\right)}\left(\frac{4}{3} t^{\frac{3}{4}}+\frac{16}{7} t^{\frac{7}{4}}+\frac{192}{77} t^{\frac{11}{4}}+\frac{768}{385} t^{\frac{15}{4}}\right)\right)+\left(-\frac{1}{\sqrt{\pi}}\left(\frac{4 t^{\frac{3}{2}}}{3}+\frac{8 t^{\frac{5}{2}}}{5}+\frac{48 t^{\frac{7}{2}}}{35}+\frac{32 t^{\frac{9}{2}}}{35}\right)\right) \\
& -\frac{\sqrt{\pi}}{\Gamma\left(\frac{3}{4}\right)^{2} \Gamma\left(\frac{1}{4}\right)}\left(\frac{256 t^{\frac{9}{4}}}{45}+\frac{4096 t^{\frac{13}{4}}}{819}+\frac{373760 t^{\text {fra } 21}}{51051}+\frac{524288 t^{\frac{21}{4}}}{54145}+\frac{116391936 t^{\frac{25}{4}}}{14889875}\right) \\
& -\frac{\sqrt{\pi}}{\Gamma\left(\frac{3}{4}\right)^{2} \Gamma\left(\frac{1}{4}\right)}\left(\frac{1509949446 t^{\frac{29}{4}}}{33215875}+\frac{603976776 t^{\frac{33}{4}}}{365374625}\right)+\ldots \\
& =u(t)
\end{aligned}
$$




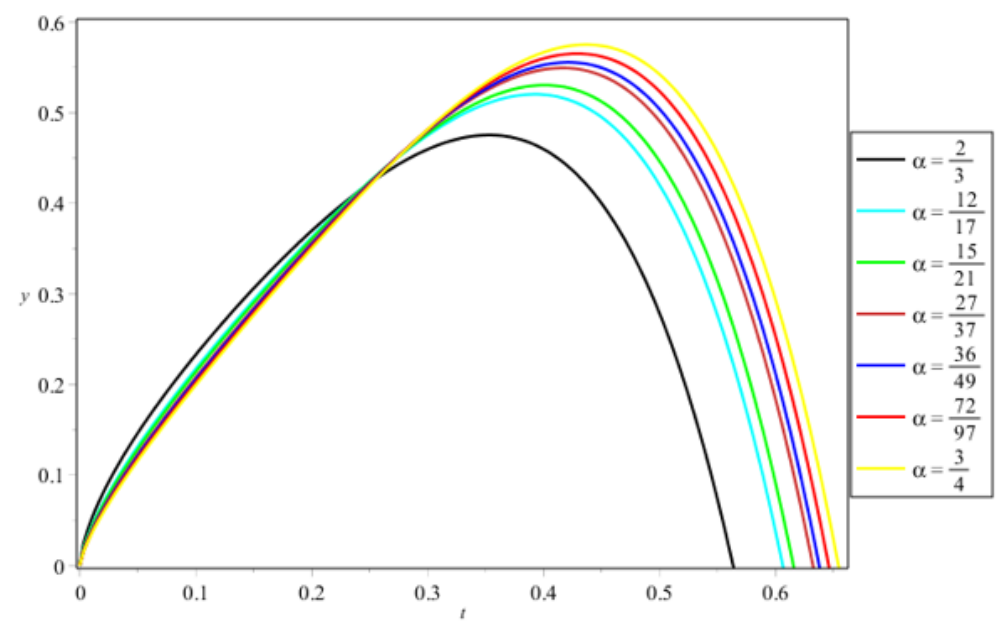

Gambar 2. Grafik fungsi solusi $D^{\alpha} u(t)+A \cdot u^{2}(t)+B \cdot u(t)=e^{k t}$ orde $\left(\alpha_{n}\right)=\left(\frac{3 n}{4 n+1}\right)$

Berdasarkan Gambar 2, solusi dikerjakan sampai iterasi suku ke-2, dapat dilihat bahwa persamaan diferensial fraksional non-linear berorde $\left(\alpha_{n}\right)$ menghasilkan pola grafik yang bergerak menuju persamaan diferensial fraksional non-linear berorde $\alpha=\frac{3}{4}$, sehingga barisan fungsi solusi persamaan diferensial fraksional non-linear dengan bentuk $D^{\left(\frac{3 n}{4 n+1}\right)} u(t)+u^{2}(t)+$ $u(t)=e^{3 t}$ konvergen ke fungsi solusi persamaan diferensial fraksional non-linear dengan bentuk $D^{\frac{3}{4}} u(t)+u^{2}(t)+u(t)=e^{3 t}$.

3.3. Perbandingan Telescoping Decomposition Method dan Adomian Decomposition Method. Diberikan persamaan diferensial fraksional non-linear berorde $\alpha$ berikut

$$
D^{\alpha} u(t)+u^{2}(t)=1
$$

Untuk $\alpha=1$ dengan menggunakan Telescoping Decomposition Method (TDM) diperoleh solusi pendekatan sebagai berikut

$$
u_{T D M}=t-\frac{t^{3}}{3}+\frac{2 t^{5}}{5}-\frac{17 t^{7}}{63}+\frac{62 t^{9}}{2835} .
$$

Untuk $\alpha=1$ dengan menggunakan Adomian Decomposition Method (ADM) diperoleh solusi pendekatan sebagai berikut

$$
u_{A D M}=t-\frac{t^{3}}{3}+\frac{2 t^{5}}{15}
$$

Untuk $\alpha=1$ diperoleh solusi eksak sebagai berikut

$$
u(t)=\frac{e^{2 t}-1}{e^{2 t}+1}
$$

Solusi pendekatan untuk persamaan (11) dengan menggunakan Telescoping Decomposition Method (TDM) lebih cepat kekonvergenannya menuju solusi eksak dibanding dengan menggunakan Adomian Decomposition Method (ADM).

\section{SIMPULAN}

Solusi persamaan diferensial fraksional non-linear dengan TDM selalu ada untuk $0<$ $\alpha \leq 2$, dan jika $\left(\alpha_{n}\right)$ konvergen ke $\alpha$ maka barisan fungsi solusi dari persamaan diferensial dengan koefisien fungsi berorde $\alpha_{n}$ akan konvergen ke fungsi solusi persamaan diferensial fraksional dengan koefisien fungsi berorde $\alpha$. TDM terbukti lebih efisien dibandingkan ADM dalam 


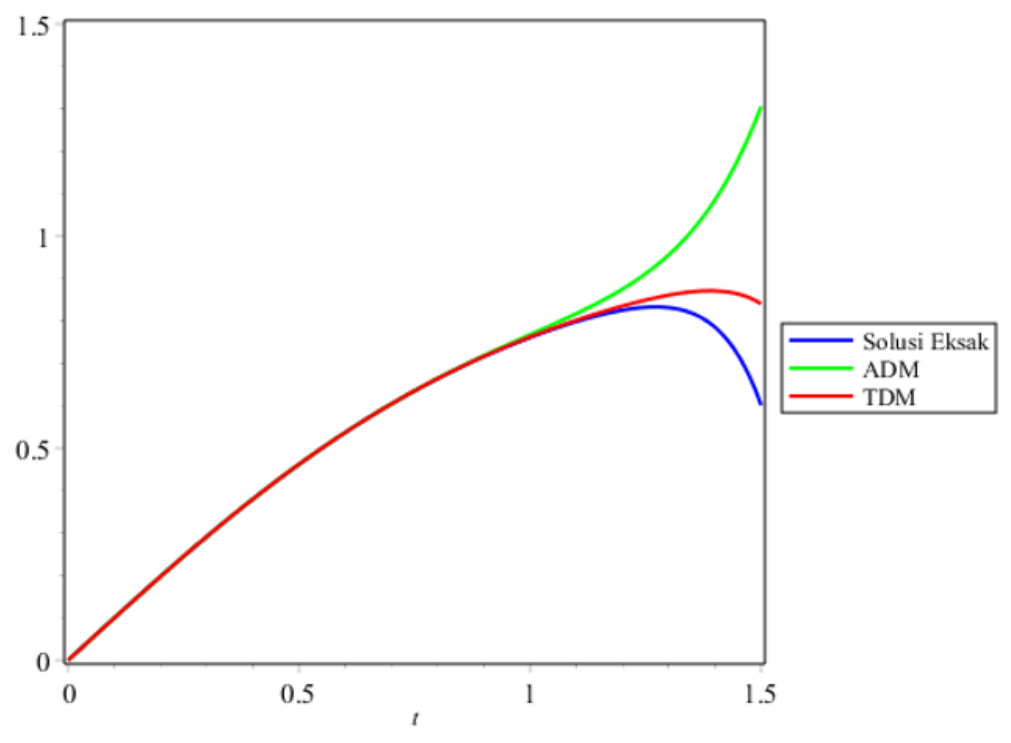

Gambar 3. Grafik solusi $D^{\alpha} u(t)+u^{2}(t)=1$ dengan $\alpha=1$

mencari solusi karena tidak perlu mencari polinomial adomian terlebih dahulu, serta kekonvergenan barisan fungsi solusi dengan menggunakan TDM lebih cepat konvergen menuju solusi eksak dibandingkan dengan menggunkan ADM tetrlihat pada Gambar 3.

4.1. Saran. Artikel ini terbatas hanya pada menyusun algoritma dan menentukan bilangan kromatik fuzzy dari graf fuzzy secara simulasi dengan bantuan software MATLAB. Saran untuk penelitian berikutnya adalah menerapkan algoritma ke dalam penyusunan jadwal perkuliahan.

\section{Daftar Pustaka}

[1] Al-Refai, Mohammed. et al. 2008. Telescoping Decomposition Method for Solving First Order Nonlinear Differential Equations. Vol 11, 19-21.

[2] Nibron, M.H. et al. 2014. Telescoping Decomposition Method for Solving Second Order Nonlinear Differential Equations. Nigeria. Vol. 3, 590-595.

[3] Bouhassoun, Abdelkader. 2013. Multistage Telescoping Decomposition Method for Solving Fractional Differential Equations. Vol. 43, 10-16.

[4] Kisela, Thomas. 2008. Mathematical Methods in The Physical Sciences. Thesis Faculty Of Mechanical Engineering, Institute Of Mathematics, BRNO University Of Technology. 